\title{
History of alcohol abuse reduces response to antipsychotics in people with first episode psychosis
}

Green Al, Tohen MF, Hamer RM, et al. First episode schizophrenia-related psychosis and substance use disorders: acute response to olanzapine and haloperidol. Schizophr Res 2004;66:125-35.

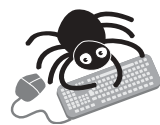

This article

contains extra text on the EBMH website

\section{What is the prevalence of substance use disorder among people with first episode psychosis, and does substance use disorder affect response to antipsychotic drugs?}

\section{METHODS}

口

Design: Randomised controlled trial.

Q2

Allocation: Unclear.

Blinding: Double blind

Follow up period: 12 week acute phase followed by a 92 week continuation phase (total 2 years).

Setting: Fourteen academic medical centres in North America and Western Europe (part of a larger trial comparing olanzapine $\checkmark$ haloperidol).

Patients: 262 people aged 16-40 years, with schizophrenia schizophreniform or schizoaffective disorder (DSM-IV), presenting with first episode psychosis. Participants scored $\geqslant 4$ on the Positive and Negative Syndrome Scale (PANSS) and had a Clinical Global Impression (CGI) severity score $\geqslant 4$. Exclusions: substance use in previous month, received antipsychotic drugs for more than 16 weeks, unstable medical illness, or high risk of suicide.

RX Intervention: Olanzapine $(5-20 \mathrm{mg} /$ day) or haloperidol (2-20 mg/day), titrated over 12 weeks. Chloral hydrate or diazepam for a maximum of 21 days was permitted. Antiparkinsonian drugs were allowed as necessary. Participants were assessed at baseline for alcohol, cocaine, cannabis, hallucinogen, and substance use disorders (SUD).

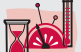

Outcomes: Psychopathology assessed using established scales (PANSS, MADRS, CGI severity item) measured weekly from weeks $0-6$ and then biweekly for weeks 7-12. Substance use assessed with SCID.

Patient follow up: Substance use assessment: 100\%; 12 week acute phase 60\%; analysis: last observation carried forward.

\section{MAIN RESULTS}

Prevalence of SUD: $37 \%$ had a lifetime SUD diagnosis, most commonly with cannabis $(38 \%)$, then alcohol $(21 \%)$, cocaine $(6 \%)$, hallucinogens/ PCP $(5 \%)$, and opioids (1\%). People with SUD were more likely to be male and have had longer periods of untreated psychosis than their non-SUD counterparts. Response to antipsychotics: people with alcohol use disorder (AUD) were significantly less likely to respond compared with people without AUD; differences in response were not significant for people with or without SUD or cannabis use disorders (see http:// www.ebmentalhealth.com/supplemental for table). People with AUD were less likely to respond to olanzapine compared with haloperidol $(9 \% \vee 27 \%, \mathrm{p}<0.02)$.

For correspondence: Alan I Green, Dartmouth Medical School, One Medical Center Drive, Lebanon, NH, USA; Alan.I.Green@Dartmouth.edu

Sources of funding: USPHS grants and by Lilly Research Laboratories.

\section{CONCLUSIONS}

A history of substance use disorder is likely in people presenting with first episode psychosis. This may reduce their response to antipsychotic medication.

\section{Commentary}

Ithough this study did not test the efficacy of haloperidol and olanzapine when SUD were concurrently present in people with psychoses, it did confirm the often reported finding that a history of substance misuse is a significant predictor of poorer therapeutic response. Both typical and novel neuroleptics showed comparable efficacy in reducing psychotic symptom scores in patients without a history of SUD. Olanzapine appeared be less effective than haloperidol in people with a history of SUD; particularly those with comorbid alcoholism. This is a puzzling finding. There is a growing consensus in the addiction psychiatry field that favours atypical antipsychotics to treat such dual pathology. ${ }^{2}{ }^{3}$ Olanzapine had been found effective in reducing both psychotic and addiction symptoms in previous open label ${ }^{4}$ and naturalistic follow up studies. ${ }^{5}$

Preference for atypicals is based on some plausible assumptions: (1) typical neuroleptics, with their prolonged blockage of D2 receptors, are more likely to induce hypersensitisation with a consequent increase in drug reward effects ${ }^{6} ;(2)$ they cause parkinsonian depletion (anhedonia) and do not improve negative symptoms, two phenomena believed to foster drug use; and (3) the improvement in cognitive function atypical antipsychotics purportedly exert increases the benefits of addiction rehabilitation therapy. ${ }^{7}$

This trial measured initial effectiveness in the early phase of psychotic illness as participants were in remission, the dual diagnosis cases it included are likely to have had less severe forms of addiction pathology. Perhaps the alleged advantages of novel antipsychotics become more evident in the chronic stages, when the cumulative effects of progressive deterioration and neuroleptic side effects are more of a factor.

This short trial has nonetheless shown, once more, that atypicals enhance treatment compliance. This is certainly an important consideration, for there is evidence that a more comprehensive therapeutic response in cases of dual pathology can only be expected in the longer term. ${ }^{8}$ On the other hand, information is still lacking on the specific metabolic effects (for example, blood lipids and glucose) that atypical neuroleptics could have in patients with liver dysfunction from alcohol damage or injection acquired viral infections.

Juan C Negrete, MD, FRCP(C) McGill University, Montreal, Canada

1 Negrete JC. Clinical aspects of substance abuse in persons with schizophrenia. Can J Psychiatry 2003;48:14-21.

2 Krystal JH, D'Souza DC, Madonick S, et al. Toward a rational pharmacotherapy of comorbid substance abuse in schizophrenic patients. Schizophrenia Res 1999;35(suppl 6):S35-S49.

3 Noordsy DL, Green Al. Pharmacotherapy for schizophrenia and cooccurring substance use disorders. Curr Psychiatry Reports 2003;5:340-6.

4 Littrell KH, Petty RG, Hilligoss NM, et al. Olanzapine treatment for patients with schizophrenia and substance abuse. J Substance Abuse Treatment 2001;21:217-21.

5 Noordsy DL, O'Keefe C, Mueser KT, et al. Six-month outcomes for patients who switched to Olanzapine treatment. Psychiatr Serv 2001;52:501-7.

6 Kosten TA. Enhanced neurobehavioural effects of cocaine with chronic neuroleptic exposure in rats. Schizophrenia Bulletin 1997;23:203-13.

7 George TP, Ziedonis DM, Feingold A, et al. Nicotine transdermal patch and atypical antipsychotic medications for smoking cessation in schizophrenia. Am J Psychiatry 2000;157:1835-42.

8 Drake RE, Xie H, McHugo Gl, ef al. The effects of clozapine on alcohol and drug use disorder among patients with schizophrenia. Schizophrenia Bulletin 2000;26:441-9. 